\title{
Toxicity of Low-dose Graphene Oxide Nanoparticles in an in-vivo Wild Type of Caenorhabditis elegans Model
}

\author{
Ming-Hsien Tsai ${ }^{1}$, How-Ran Chao ${ }^{2,3,4^{*}}$, Jheng-Jie Jiang ${ }^{5,6}, \mathrm{Yu}-\mathrm{Hsieh} \mathrm{Su}^{2}$ \\ Mariene-syne P. Cortez ${ }^{7}$, Lemmuel L. Tayo ${ }^{7}$, I-Cheng Lu ${ }^{2}$, Hao Hsieh², \\ Chih-Chung Lin $^{2}$, Sheng-Lun Lin ${ }^{8,9}$, Wan Nurdiyana Wan Mansor ${ }^{10,11}$, \\ Ching-Kai Su ${ }^{12}$, Sen-Ting Huang ${ }^{13}$, Wen-Li Hsu ${ }^{4,13}$
}

\footnotetext{
${ }^{1}$ Department of Child Care, National Pingtung University of Science and Technology, Neipu, Pingtung 91201, Taiwan

2 Department of Environmental Science and Engineering, National Pingtung University of Science and Technology, Neipu, Pingtung 91201, Taiwan

${ }^{3}$ Institute of Food Safety Management, College of Agriculture, National Pingtung University of Science and Technology, Neipu, Pingtung 91201, Taiwan

${ }^{4}$ Emerging Compounds Research Center, General Research Service Center, National Pingtung University of Science and Technology, Neipu, Pingtung 91201, Taiwan

${ }^{5}$ Department of Environmental Engineering, Chung Yuan Christian University, Taoyuan 320314,

Taiwan

${ }^{6}$ Center for Environmental Risk Management, Chung Yuan Christian University, Taoyuan 320314, Taiwan

${ }^{7}$ School of Chemical, Biological and Materials Engineering and Sciences, Mapúa University, Muralla St., Intramuros, Manila 1002, Philippines

${ }^{8}$ School of Mechanical Engineering, Beijing Institute of Technology, Beijing 100081, China

${ }^{9}$ Center for Environmental Toxin and Emerging-contaminant Research, Cheng Shiu University, Kaohsiung 83347, Taiwan

${ }^{10}$ Faculty of Ocean Engineering Technology \& Informatics, Universiti Malaysia Terengganu, 21300, Malaysia

${ }^{11}$ Air Quality and Environment Research Group, Universiti Malaysia Terengganu, 21300, K. Nerus, Malaysia

${ }^{12}$ Department of Internal Medicine, Kaohsiung Veterans General Hospital Pingtung Branch, Neipu, Pingtung 91245, Taiwan

${ }^{13}$ Research Institute for Life Support Innovation, Research Organization for Nano and Life Innovation, Waseda University, Shinjuku, Tokyo 162-8480, Japan
}

Revised: November 3, 2020

Accepted: December 6, 2020

${ }^{*}$ Corresponding Author:
hrchao@mail.npust.edu.tw

Publisher:

Taiwan Association for Aerosol Research

ISSN: $1680-8584$ print

ISSN: 2071-1409 online

Copyright: The Author(s). This is an open access article distributed under the terms of the Creative Commons Attribution License (CC BY 4.0), which permits unrestricted use, distribution, and reproduction in any medium, provided the original author and source are cited.

\section{ABSTRACT}

Carbon-based engineered nanomaterials, such as graphene oxide nanoparticles (GO NPs), are widely available for application, but their potentially adverse health effects on humans still require investigation. In this study, the environmental levels of GO NPs are addressed to examine whether GO leads to adverse effects on an in-vivo model of Caenorhabditis elegans (C. elegans). Nematodes with prolonged exposure (L1 larvae to young adult) to GO NPs at 0.00100, 0.0100, 0.100 , and $1.00 \mu \mathrm{g} \mathrm{L}^{-1}$ were used to evaluate the potential toxic effects, including lethality (acute toxicity), reproductive (brood size) and neurological (locomotion including head thrash and body bend) responses, longevity (lifespan), and oxidative stress (gene expression of sod-1, sod-3, and clt-2). Prolonged exposure to GO NPs was not found to induce lethality at the selective levels. In the brood-size and head-thrash tests, the biological responses in nematodes were significantly reduced at $0.0100-1.00 \mathrm{ng} \mathrm{L}^{-1} \mathrm{GO}$ NP exposure as compared with the untreated control. The nematodes exposure to GO NPs at $0.00100-1.00 \mathrm{ng} \mathrm{L}^{-1}$ exhibited significant delays in body bending behavior compared with the control. In the examination of the longevity of nematodes, 
it was found that the lifespan of all GO NP-exposed worms was significantly shortened as compared to the untreated worms. Gene expression of sod-1, sod-3, and ctl-2 presented significantly higher induction folds in the exposed worms compared with the controls. Consequently, prolonged exposure to the low-dose GO NPs might be associated with disruption of reproduction and locomotion, attenuation of longevity, and induction of oxidative stress in nematodes.

Keywords: Graphene oxide, Caenorhabditis elegans, Reproductive toxicity, Neurobehavioral toxicity, Oxidative stress

\section{INTRODUCTION}

Graphene oxide nanoparticles (GO NPs), one of the most promising derivatives of graphene, comprise a monolayered engineered nanomaterial (ENM) with high oxygen-containing functional groups such as carboxyl, epoxy, carbonyl, and hydroxyl (Bianco et al., 2013; Li et al., 2015). Graphene oxide (GO) is known to have excellent dispersibility in many solvents, chemical reactivity, and the capacity for chemical functionalization (Konios et al., 2014; De Marchi et al., 2018). The adsorption capacity of GO NPs has been taken advantage of for environmental remediation applications such as in GO-based membranes, which remove gaseous contaminants such as sulfur dioxide and hydrogen sulfide (Fakhri, 2017). In addition, they also act as adsorbents for elimination of various aqueous contaminants owing to their high content of functionalized oxygen groups available to interact with metal ions (Li et al., 2019). Furthermore, GO NPs can be applied in nanoelectronics, catalysis, nanocomposites, sensor technology, water purification and desalination, and drug delivery (Zhang et al., 2011; Pan et al., 2012; Giust et al., 2018; Prasad et al., 2020). According to studies from Yang et al. (2015) and Maharubin et al. (2016), GO can be utilized for hydrogen storage (anode, cathode, and lithium sulfur batteries) and supercapacitor management. Furthermore, GO composites are used as antimicrobial agents for water disinfection to remove organic molecules and waterborne pathogens (Upadhyay et al., 2014). Several studies used the environmental levels at $\mu \mathrm{g} \mathrm{L}^{-1}$ to $\mathrm{mg} \mathrm{L}^{-1}$ of GO NP contamination to test the in-vivo models (He et al., 2017; Zhang et al., 2017; Li et al., 2019).

The global market for graphene-based products, such as GO, is increasing. The demand is expected to be $\$ 675$ and 987 million by 2020 and 2022, respectively (Ahmed and Rodrigues, 2013). Due to their potential for both production and application, GO materials are expected to be released in the environment during their lifecycle and eventually be generated in landfills and wastewater treatment plants (Du et al., 2017; Suárez-Iglesias et al., 2017; Jamialahmadi et al., 2018). GO can be released into the water environment through the development of its composites as adsorbents for aqueous contamination, membranes for water filtration and purification, and catalysts for environmental decontamination (Zhao et al., 2014; Goodwin et al., 2018). Several studies have reported that the dispersion and long retention time of $\mathrm{GO}$ within microbial communities can lead to serious negative effects on wastewater microbial flora due to its hydrophility (Lyon and Alvarez, 2008; Kang et al., 2009; Rodrigues and Elimelech, 2010). The predicted environmental concentrations of GO can be correlated with those of multi-walled carbon nanotubes because both have relatively similar properties, such as nanometer size, a carbon-based structure, and applications in consumer electronic devices (Zhang et al., 2017).

Few epidemiological studies focused on human exposure to GO particularly for the highly exposed population. For the in-vivo models including rats, mice, zebra fish, nematodes, and daphnia, the animals could induce nanotoxicity including acute, developmental, neurological, reproductive, immunological, and neurobehavioral toxicity as well as shortened longevity after they were exposed to GO NPs (Sanchez et al., 2012; Patlolla et al., 2017; Qu et al., 2017; Souza et al., 2017; Kim et al., 2018; Qu et al., 2019; Kim et al., 2020). In the past years, in vivo and in vitro GO NPs toxic effects, including immunotoxicity, activation of inflammation, induction of reactive oxygen species (ROS), generation of oxidative stress, apoptosis, and potential GO exposure mechanisms have been investigated (Guo and Mei, 2014; Bengtson et al., 2017; Pelin et al., 2018; Tang et al., 2018). The accumulation of $\mathrm{GO}$ in the cytoplasm causes dramatic morphological alterations and reduces the ability of toll-like receptor 4 (TLR4) for phagocytosis (Qu et al., 2013a). However, an increase in intracellular ROS contributes to necrotic cell death in macrophages (Qu et al., 2013a). Previous 
studies have also reported that GO promotes cell growth inhibition, hatching delay, ROS generation, and damages the circulatory system of zebrafish embryos (Liu et al., 2014; Chen et al., 2016; Souza et al., 2017). In mice, GO can accumulate in organs such as the liver, lungs, spleen, and kidneys, which may induce organismal toxicity through intracellular oxidative stress caused by the accumulation of ROS (Qu et al., 2013b; Yang et al., 2013). GO can enter the human body through inhalation and may be deposited in regions of the respiratory tract. When deposited in alveolar regions, it may impair clearance, form granulomas, and possibly produce fibrosis (Sanchez et al., 2012).

The in vivo model used in this study was the transparent nematode, Caenorhabditis elegans (C. elegans), which has been successfully used in toxicological evaluation of various nanomaterials such as GO NPs (Zhang et al., 2012; Wu et al., 2013; Piechulek and von Mikecz, 2018). Advantages of $C$. elegans as an in-vivo model system were as the following: (1) simple anatomy, (2) transparent, (3) invariant cell lineage, (4) short life cycle with large brood size, (5) easily accessible embryos, (6) easy and cheap maintenance in lab, and (7) powerful experimental tool (Brenner, 1974; Hunt, 2017). The $C$. elegans model is not a mammal model to unavailable to examine several toxic endpoints like blood sugar and pressure, tissues in the skin, probiotic system in the intestine, heart and cardiovascular diseases. C. elegans is considered to be a novel tool for in-vivo techniques, and testing of $C$. elegans is known to be analogous to mammalian neurotoxin testing (Cole et al., 2004). Recently, scientists have focused on the disruption of biological effects from ROS, reproductive effects, gene expression, neurological development, and neurobehavior with treatment of GO NPs in C. elegans models (Wu et al., 2013; Qu et al., 2017; Kim et al., 2018; Rive et al., 2019; Kim et al., 2020; Zhao et al., 2020). Zhang et al. (2012) indicated no negative impact on longevity after exposing L4-larva-to-young-adult $C$. elegans to GO NPs at concentrations ranging from 5 to $20 \mathrm{mg} \mathrm{L}^{-1}$. Inversely, $C$. elegans with prolonged exposure (from L1 larva to young adult) to 0.5$100 \mathrm{mg} \mathrm{L}^{-1}$ of GO NPs presented adverse effects on primary (digestive organs such as the intestine) and secondary (neurological tissues such as neurons and reproductive organs) target organs (Wu et al., 2013). GO NPs possibly shortened lifespan by influencing the expression of the DAF-2-AGE1-AKT-1/2-DAF-16 signaling cascade in the intestine of the nematodes (Zhao et al., 2016b). After GO NP exposure, expression of neuronal substances may decrease ROS generation and reduce locomotion behavior in nematodes (Zhao et al., 2020). GO NPs probably caused damage to the dopaminergic and glutamatergic neurons in C. elegans after chronic exposure to GO NPs for 6 days, from L1 larvae to the adult stage (Li et al., 2017). Liu et al. (2020) observed that GO NPs induced intestinal barrier dysfunction in C. elegans. Rive et al. (2019) proposed that worms chronically (or prolongedly) exposed to GO NPs (levels of 100 and $200 \mathrm{mg} \mathrm{L}^{-1}$ ) were significantly shortened in size and developed morphological abnormalities in the pharynx and intestine. Kim et al. (2018) found accumulation of GO NPs in the reproductive organs of $C$. elegans using Raman spectrometry. Also, GO NP exposure promoted reproductive toxicity by suppressing spermatogenesis of C. elegans during development, resulting in decreased sperm numbers and progeny numbers (Kim et al., 2018). This study was aimed toward evaluating the effect of environmentally-relevant concentrations of GO NPs in C. elegans by assessing toxicological endpoints including acute lethality, reproduction, locomotion, lifespan, and gene expression.

\section{METHODS}

\subsection{Preparation of Graphene Oxide Nanoparticles}

GO NPs were prepared from expandable graphite using a modified Hummers' method (Yan et al., 2014). In brief, $1 \mathrm{~g}$ graphite power and $50 \mathrm{~mL}$ sulfuric acid $\left(\mathrm{H}_{2} \mathrm{SO}_{4}, 98 \%\right)$ were poured into a $250 \mathrm{~mL}$ flask, followed by the addition of $0.5 \mathrm{~g} \mathrm{NaNO}_{3}$. The mixture was mechanically agitated for $30 \mathrm{~min}$ in an ice bath. For further oxidation, $5 \mathrm{~g}$ of potassium permanganate $\left(\mathrm{KMnO}_{4}\right)$ was added while slowly stirring the mixture for $4 \mathrm{~h}$. Subsequently, $\mathrm{H}_{2} \mathrm{O}_{2}$ was added to $\mathrm{MnO}_{2}$ until the mixture became yellow. Afterward, $1 \% \mathrm{HCl}$ was added, and the mixture was centrifuged at $8000 \mathrm{rpm}$ for $5 \mathrm{~min}$, followed by washing 3 times with distilled water to dilute the acid solution.

\subsection{Reagents, Chemicals, and Nematode Cultivation}

The GO NPs underwent sonication for $30 \mathrm{~min}(40 \mathrm{kHz}$ and $100 \mathrm{~W})$ to disperse them in $\mathrm{K}$ medium (50 mM, $30 \mathrm{mM} \mathrm{KCl}, 1.0 \mathrm{mg} \mathrm{mL}^{-1}$ and $\mathrm{PH}$ of 6.0 ) as the stock solution (200 $\mathrm{mg} \mathrm{L}^{-1}$ ) following the 
methods in previous studies (Wu et al., 2013; Zhao et al., 2016b). The stock solution was diluted to various concentrations using $\mathrm{K}$ medium prior to exposure.

The wild-type N2 C. elegans strain was gifted from Dr. Chang-Shi Chen in the Department of Biochemistry and Molecular Biology, College of Medicine, National Cheng Kung University (Tainan, Taiwan). C. elegans was maintained on nematode growth medium (NGM) seeded with OP50 Escherichia coli (E. coli) cultures from the Bioresources Collection and Research Center (Hsinchu, Taiwan), and Luria-Bertani broth was obtained from Sigma-Aldrich (St. Louis, MO, USA). The NGM plates contained bacteriological agar and bactopeptone, which were obtained from Laboratories Conda (S.A., Spain). The $\mathrm{NaCl}$ was obtained from Honeywell Fluka ${ }^{\mathrm{TM}}$ (New Jersey, USA). Agesynchronized worms were collected using a bleaching mixture that contained $\mathrm{NaOCl}$ obtained from J.T. Baker (Central Valley, PA) and KOH obtained from Duksan Pure Chemicals (Gyeonggido, South Korea). Supplemental reagents such as $\mathrm{CaCl}_{2}, \mathrm{~K}_{2} \mathrm{HPO}_{4}$, and cholesterol were obtained from Sigma-Aldrich (St. Louis, $\mathrm{MO}, \mathrm{USA}$ ); $\mathrm{MgSO}_{4}$ was acquired from Avantor Performance Materials, Itd. (Gyeonggi-do, South Korea); the $\mathrm{KH}_{2} \mathrm{PO}_{4}$ used for the phosphate buffer was acquired from Avantor Performance Materials, LLC (Radnor, PA, USA), and the $\mathrm{Na}_{2} \mathrm{HPO}_{4}$ used for the M9 buffer was obtained from Honeywell Fluka ${ }^{\mathrm{TM}}$ (New Jersey, USA). All physiological observations were done under a dissecting microscope (Olympus, SZX10, Waltham MA, USA). The experimental protocols in the nematode model followed those used in our previous study (Chung et al., 2019, 2020). The lethality, growth, reproduction, locomotion behavior examinations followed the protocols previously published, with minor modifications (Chung et al., 2019, 2020).

\subsection{Lethality and Lifespan Assay}

The nematodes were exposed to GO NPs (control, 0.00100, 0.0100, 0.100, and $1.00 \mu \mathrm{g} \mathrm{L}^{-1}$ ) for $48 \mathrm{~h}$ (prolonged exposure) from L1-larvae to young adults incubated at $20^{\circ} \mathrm{C}$. Prolonged exposure was performed in a fresh plate with an OP50 E. coli lawn. After treatment, the lethal toxicity of the samples was evaluated by softly poking them using a worm picker. The worms that did not respond were considered dead. Three biological replicates were performed, and a total of 150 worms were assayed.

The worms evaluated for the lifespan assay were exposed for a prolonged period of time to the different GO NP concentrations (the untreated control, 0.00100, 0.0100, 0.100, and $1.00 \mu \mathrm{g} \mathrm{L}^{-1}$ ) from $\mathrm{L} 1$ to the mature stage for the lifespan test. Fifty worms were transferred to fresh plates every other day for 4-5 days of egg-laying. Live and dead nematodes were evaluated daily by softly poking them with a worm picker. Three biological replicates were performed, and a total of 150 worms were evaluated. Several lifespan indicators (mean lifespan, day of $50^{\text {th }}$ percentile death, day of $75^{\text {th }}$ percentile death, day of $95^{\text {th }}$ percentile death, and day of all death) were evaluated by following the Chung's study (Chung et al., 2020).

\subsection{Reproductive Assay and Locomotion Assay}

The brood size (reproductive assay) of the L3 or young L4 nematodes was assessed for 4-5 days after prolonged exposure to different GO NP concentrations (control, 0.00100, 0.0100, 0.100, and $1.00 \mu \mathrm{g} \mathrm{L}^{-1}$ ) at $20^{\circ} \mathrm{C}$. Each worm was transferred to a fresh plate and transferred again until the egg-laying period stopped. The plates with eggs were incubated until the progeny could be easily counted. A total of 30 worms was evaluated for the reproductive assay.

Locomotion behavior including head thrashing and body bending in the nematode models was expressed as the motor neuron function (Qu et al., 2019; Zhao et al., 2020). The body bending and head thrashing of the nematodes (locomotion assay) were evaluated after prolonged exposure to various concentrations of GO NP (control, $0.00100,0.0100,0.100$, and $1.00 \mu \mathrm{g} \mathrm{L}^{-1}$ ). The body bending was evaluated by transferring the exposed worm onto a fresh plate. After one day, the body bending of the worms was counted for 20 secs. The head thrashing was evaluated by placing the exposed worm on a glass slide containing an adequate amount of K-media. The head thrashing of the worms was counted for $1 \mathrm{~min}$. Three biological replicates were performed, where 60 worms were evaluated for body bending, and 30 worms were evaluated for head thrashing.

\subsection{Gene Expression Tests}

C. elegans in the different treatment groups (untreated control, $0.00100,0.100$, and $1.00 \mu \mathrm{g} \mathrm{L}^{-1}$ ) 
were collected from three replicates for RNA extraction after exposure. Trizol reagent (TIANGEN, China) was used to extract total RNA in accordance with the manufacturer's standard protocol. RNA concentrations were measured by the absorbance at $260 \mathrm{~nm}$, and the RNA purity was evaluated based on the ratio of the optical densities from RNA samples measured at 260 and $280 \mathrm{~nm}$. The first-strand cDNA synthesis reaction was conducted with $500 \mathrm{ng}$ of purified RNA using a Fast Quant RT Kit (with gDNase) according to the manufacturer's protocol (TIANGEN, China). Specific superoxide dismutase genes, including (sod) 1 (sod-1), sod-3, and catalase 2 (ctl-2) were detected in the present study. The data were analyzed using the $2-\Delta \Delta \mathrm{Ct}$ method, as previously reported (Zhou et al., 2016), and the mRNA expressions were normalized based on the act-1 mRNA. For each tested gene, a qRT-PCR analysis was conducted in triplicate (technical replicates).

\subsection{Statistical Analysis}

The Statistical Package for the Social Sciences (SPSS) version 12 software (International Business Machines Corp., New York, USA) was used to perform all statistical analyses. All data was checked to normality, and the Shapiro-Wilk test was used determined the normal and nonnormal distribution. A one-way ANOVA was used to analyze the significance levels of the differences between treatments. The plots and figures were made using GraphPad Prism 6 (San Diego, California, USA).

\section{RESULTS AND DISCUSSION}

\subsection{GO NPs Lethality}

C. elegans was evaluated through a variety of toxicological endpoints, including lethality, reproduction, locomotion, lifespan, and oxidative stress (gene expression of sod-1, sod-3, and clt-2) in the present study. This study is the first time that low doses of GO NP (approximately at least 1000-fold lower compared to those used in the previous studies) has been used to examine nanotoxicity in a $C$. elegans model. Exposure from L1-larvae to young adult was performed to assess the effects of prolonged GO exposure on both larvae and adult nematodes. As shown in Fig. 1, the survival rate of the nematodes after prolonged exposure to GO did not indicate significant lethal effects. No significant between-group differences in mortality were observed in the

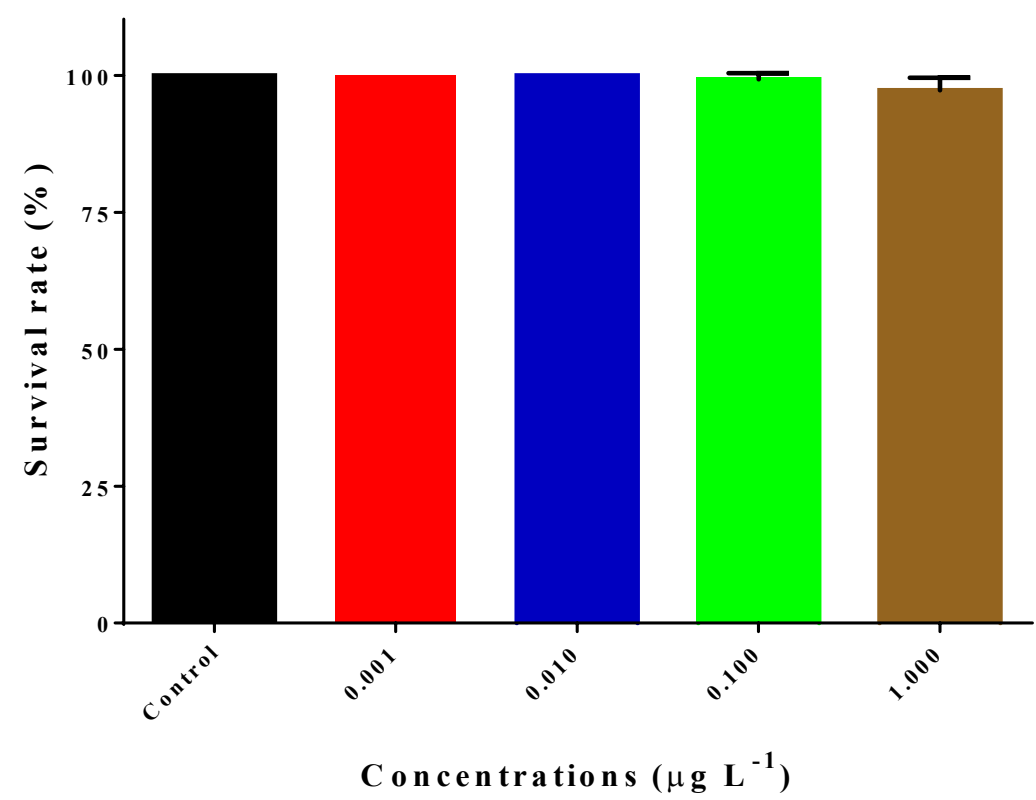

Fig. 1. Survival rates of $C$. elegans after prolonged exposure to GO NPs at concentrations of $0.00100,0.0100,0.100$, and 1.00 and the untreated control. Bars shown as mean \pm SD. Significant differences were expressed as $* p<0.05,{ }^{* *} p<0.01$, and $* * * p<0.001$. 
control group and in the group treated with concentrations ranging from 0.00100 to $1.00 \mu \mathrm{g} \mathrm{L}^{-1}$. Few studies have examined acute toxicity of GO NPs in $\mathrm{N}_{2}$ C. elegans models (Wu et al., 2013, 2014; Li et al., 2019). Wu et al. (2013) indicated that no lethality was observed at GO NP concentrations from 0.100 to $100 \mathrm{mg} \mathrm{L}^{-1}$ after the worms were acutely or prolongedly exposed to these carbon-based ENMs. On the contrary, a study examining acute toxicity with treatment of high doses of $5,10,50$, and $100 \mathrm{mg} \mathrm{L}^{-1}$ of GO NPs in nematodes found that GO concentrations higher than $5 \mathrm{mg} \mathrm{L}^{-1}$ may cause lethality, where no worms survived at a dosage of $100 \mathrm{mg} \mathrm{L}^{-1}$ ( $\mathrm{Li}$ et al., 2017). Wu et al. (2014) also examined low levels of GO NPs from 0.00100 to $1.00 \mathrm{mg} \mathrm{L}^{-1}$ and found no significant differences in lethality except in the case of the highest concentration of $1.00 \mathrm{mg} \mathrm{L}^{-1}$ after the nematodes were chronically exposed to these carbon-based ENMs from L1 larvae to adult-day 8. Most GO NP studies refer to Wu's study (Wu et al., 2013) and use similar dosage levels (ppm levels) to examine neurological, reproductive, neurobehavioral, and immunological toxicity, and inflammatory responses.

\subsection{Reproductive Toxicity of GO NPs}

Reproduction in nematodes is a vital endpoint because it has been shown to be sensitive to lower concentrations of chemical stressors than those that impair the behavior and viability of nematodes (Wu et al., 2019). The results in Fig. 2 show that prolonged exposure to GO NPs in the nematodes reduced brood size production. A significant decrease was observed in the progeny number at concentrations of $0.0100(p=0.036), 0.100(p=0.008)$, and $1.00(p<0.001)$ $\mu \mathrm{g} \mathrm{L}^{-1}$ of GO NPs. The reduction rates in the brood size at these three concentrations compared to the control group were 15.2, 20.1 and $27.3 \%$, respectively. The results showed that higher concentrations of GO NPs induced more reproductive toxicity based on our experiments on brood size number in nematodes. Our results were consistent with most GO NP studies reporting that GO exposure can cause adverse effects through damaging the fertility and egg ejection behavior of nematodes (Wu et al., 2013; Zhao et al., 2016a; Kim et al., 2018; Rive et al., 2019). Wu et al. (2013) showed that $C$. elegans with prolonged exposure to $1-100 \mathrm{mg} \mathrm{L}^{-1}$ exhibited significantly decreased brood size compared to the control, but there were no significant between-group differences at 0.1 and $0.5 \mathrm{mg} \mathrm{L}^{-1}$. A similar result was also found in a previous study (Rive et al., 2019), indicating that prolonged exposure to GO NPs at 100 and $200 \mathrm{mg} \mathrm{L}^{-1}$ significantly decreased egg-laying rates compared to the untreated control. Kim et al. (2018) revealed that accumulation of GO NPs $\left(10 \mathrm{mg} \mathrm{L}^{-1}\right)$ in the reproductive organs, which might be

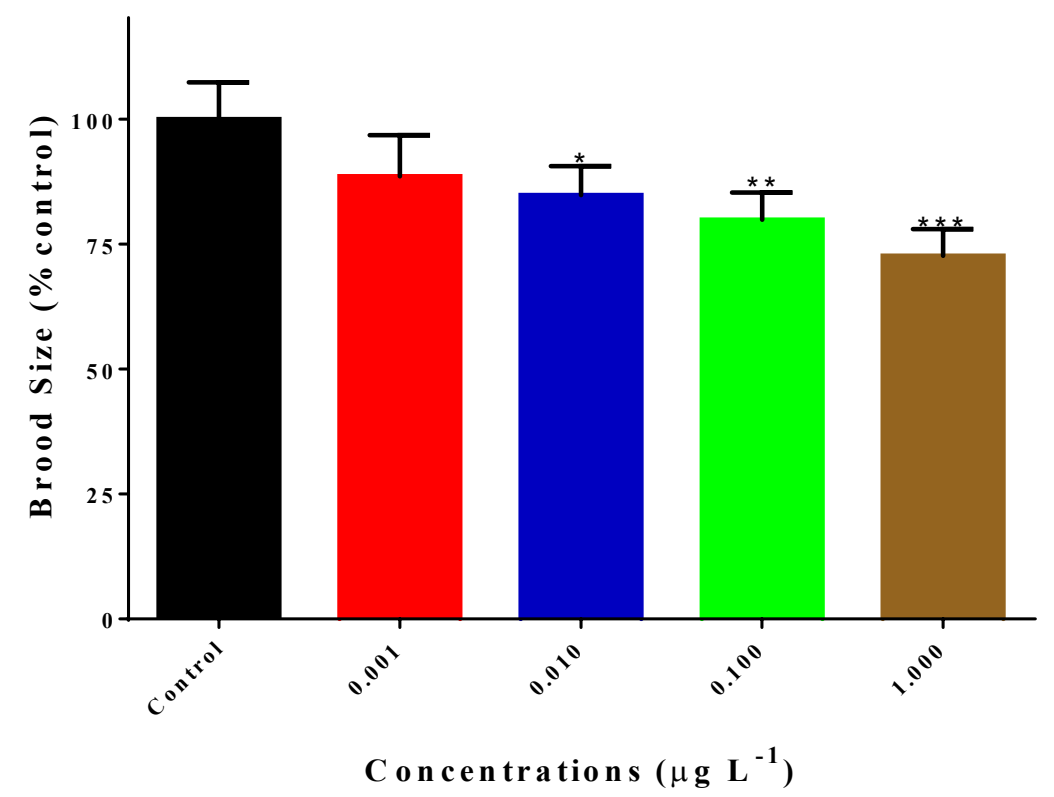

Fig. 2. Effects of the brood size in the $C$. elegans after prolonged exposure to GO NPs at concentrations of $0.00100,0.0100,0.100$, and 1.00 and the untreated control. Bars shown as mean \pm SD. Significant differences were expressed as $* p<0.05, * * p<0.01$, and ${ }^{* * *} p<0.001$. 
the direct cause of reproductive toxicity, could reduce brood size and sperm count by suppressing spermatogenesis of the hermaphrodite nematodes at the $\mathrm{GO}$ levels of 5 or $10 \mathrm{mg} \mathrm{L}^{-1}$. However, the negative impact of GO NP exposure on the reproductive function in the present and published studies (Wu et al., 2013; Zhao et al., 2016a; Kim et al., 2018; Rive et al., 2019), as well as our results, suggest that prolonged exposure to GO NPs at low doses from 0.0100 to $1.00 \mu \mathrm{g} \mathrm{L}^{-1}$ could decrease progeny number or fecundity in $\mathrm{N}_{2} \mathrm{C}$ elegans models.

\subsection{GO NP Exposure Affects Locomotive Behavior}

Locomotive behavior assays are well-established methods for studying nematode neurotoxicity. After prolonged exposure, GO induced obvious decreases in both head thrashing and body bending in nematodes (Fig. 3). In the head thrash examination, $0.0100,0.100$ and, $1.00 \mu \mathrm{g} \mathrm{L}^{-1}$ concentrations of GO NPs significantly decreased head thrashing by $12.0,5.41$, and $19.8 \%$, respectively, compared
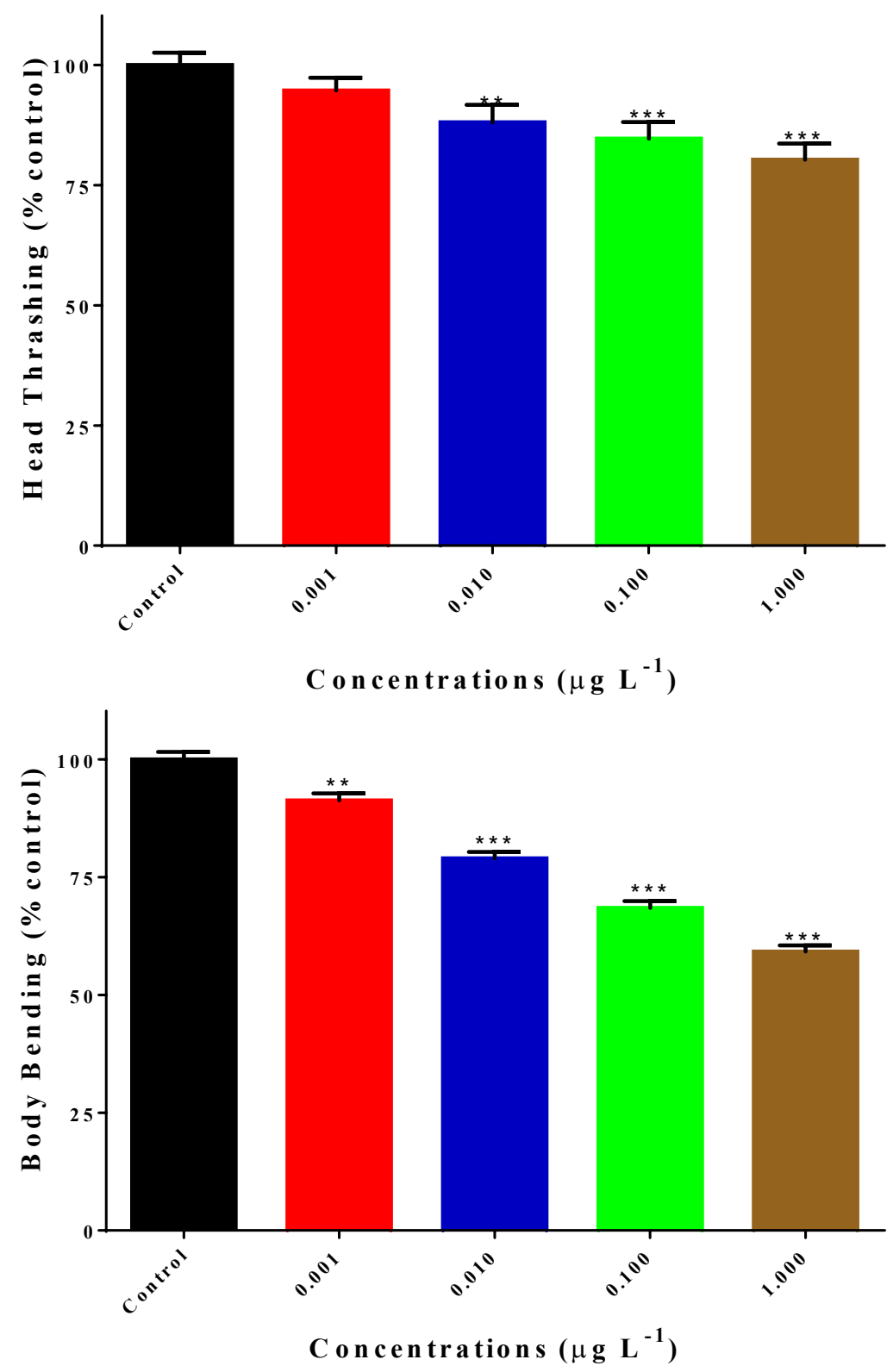

Fig. 3. Effects of head thrashing and body bending in the nematodes after prolonged exposure to GO NPs at concentrations of $0.00100,0.0100,0.100$, and 1.00 and the untreated control. Bars shown as mean \pm SD. Significant differences were expressed as $* p<0.05, * * p<0.01$, and $* * * p$ $<0.001$. 
to the untreated control. Furthermore, body bending was significantly reduced at 0.00100 , $0.0100,0.100$ and $1.00 \mu \mathrm{g} \mathrm{L}^{-1} \mathrm{GO}$ NPs by $8.78,21.2,31.5$, and $40.8 \%$, respectively, in comparison with the control groups. Our results were consistent with those in most published articles, implying that GO NP exposure damages the neurological functions and negatively disrupts head thrashing and body bending behavior (Wu et al., 2013, 2014; Zhao et al., 2015, 2016c; Chen et al., 2017; Li et al., 2017; Qu et al., 2017; Kim et al., 2018; Rive et al., 2019; Zhao et al., 2020). In Wu's report (Wu et al., 2014), head thrash and body bend locomotion was significantly reduced at $0.0100,0.100$, and $1.00 \mathrm{mg} \mathrm{L}^{-1}$ levels compared with an untreated control. Li et al. (2017) indicated that prolonged exposure to GO NPs $\left(5.00-100 \mathrm{mg} \mathrm{L}^{-1}\right)$ significantly reduced body bending, head thrashing, pharynx pumping frequency, mean speed, bending angle-frequency, and the wavelength of the crawling movement of nematodes. GO NPs also induced damage to dopaminergic and glutamatergic neurons in nematodes (Li et al., 2017). Kim et al. (2020) also proposed that GO significantly accumulated in the head regions, generated ROS induction, reduced neurotransmitter substances in dopaminergic and glutamatergic neurons, and damaged AFD neurons, which are the main thermosensors in C. elegans, after the nematodes were exposed to GO NPs (10 $\mathrm{mg} \mathrm{L}^{-1}$ ). In a Korean study, Kim et al. (2018) also found that neurotransmitters, such as dopamine, $\mathrm{y}$-Aminobutyric acid (GABA), tyramine, tryptophan, and tyrosine, were reduced in nematodes exposed to GO NPs. According to the current data, including the present study (Wu et al., 2013, 2014; Zhao et al., 2015, 2016a; Chen et al., 2017; Li et al., 2017; Qu et al., 2017; Kim et al., 2018; Rive et al., 2019; Zhao et al., 2020), it has been concluded that GO NPs exposure causes adverse effects on the neurological system of $C$. elegans particularly in terms of damage to neurons, influences on neurotransmitter neurodisruptions, and delays in neurobehavioral development. In the present study, environmental levels $\left(0.0100-1.00 \mu \mathrm{g} \mathrm{L}^{-1}\right)$ of GO NP doses were used to treat the nematodes to determine the negative impact on their locomotion behavior.

\subsection{Effect of GO NPs on Lifespan}

In C. elegans models, lifespan is an important endpoint for assessment of toxicants. After prolonged exposure in nematodes, GO NPs at concentrations of 0.00100-1.00 $\mu \mathrm{g} \mathrm{L}^{-1}$ led to shorter lifespans than was the case for the untreated controls (Fig. 4). Several indicators of lifespan, including mean lifespan (Fig. 4(b)), mean day of median ( $50^{\text {th }}$ percentile) death (Fig. $4(\mathrm{c})$ ), mean day of $75^{\text {th }}$ percentile death (Fig. 4(d)), mean day of $95^{\text {th }}$ percentile death (Fig. 4(e)), and the day of all death (Fig. 4(a)) indicated significantly longer longevity in the untreated control as compared to in GO NP-exposed nematodes $(p<0.001)$. The mean lifespan and the day of all death were $13.9,7.01,6.23,6.94$, and 6.35 days in the untreated control and $0.00100,0.0100,0.100$, and $1.00 \mu \mathrm{g} \mathrm{L}^{-1}$, respectively, and $20,16,14,14,16$ days in the untreated control and $0.00100,0.0100$, 0.100 , and $1.00 \mu \mathrm{g} \mathrm{L}-1$, respectively. After 6 days, the percent survival rate of nematodes decreased to as much as $50 \%$ of the total population. It was also observed that the nematodes treated with GO NPs exhibited faster reductions in lifespan than the control group. In summary, Fig. 4 indicates that prolonged exposure to GO NPs reduces the lifespan of nematodes $(p<0.001)$. According to the current data from previous reports (Zhang et al., 2012; Zhao et al., 2016b, 2016c; Qu et al., 2017; Rive et al., 2019), contradictory results were obtained, where two studies indicated that GO NP exposure, including both acute and prolonged exposure didn't have effects on longevity (Zhang et al., 2012; Rive et al., 2019), and other studies obtained different results indicating that the worms with prolonged exposure to GO NPs exhibited significantly reduced longevity (Zhao et al., 2016b, 2016c; Qu et al., 2017) at GO NP concentrations between 1.00 and $200 \mathrm{mg} \mathrm{L}^{-1}$. Two molecular mechanisms of intestinal insulin signaling may be involved in the shortened longevity of nematodes exposed to an GO NP concentration of $100 \mathrm{mg} \mathrm{L}^{-1}$ due to association with suppression of DAF-16 and sod-3 functions (Zhao et al., 2016b). Based on our results, the low dose of $0.00100 \mu \mathrm{g} \mathrm{L}{ }^{-1}$ significantly reduced the nematodes' longevity.

\subsection{Gene Expression after GO NPs Exposure}

The sod genes encode superoxide dismutases (SODs), which comprise an antioxidant system for $C$. elegans against oxidative stress after GO NP exposure (Ren et al., 2018). SODs which exist in three isoforms of sod1, sod2, and sod-3 are a class of the antioxidant protein. The increased folds in expression from the induced sod-1, sod-3, and ctl-2 genes after $C$. elegans had undergone 
(a)
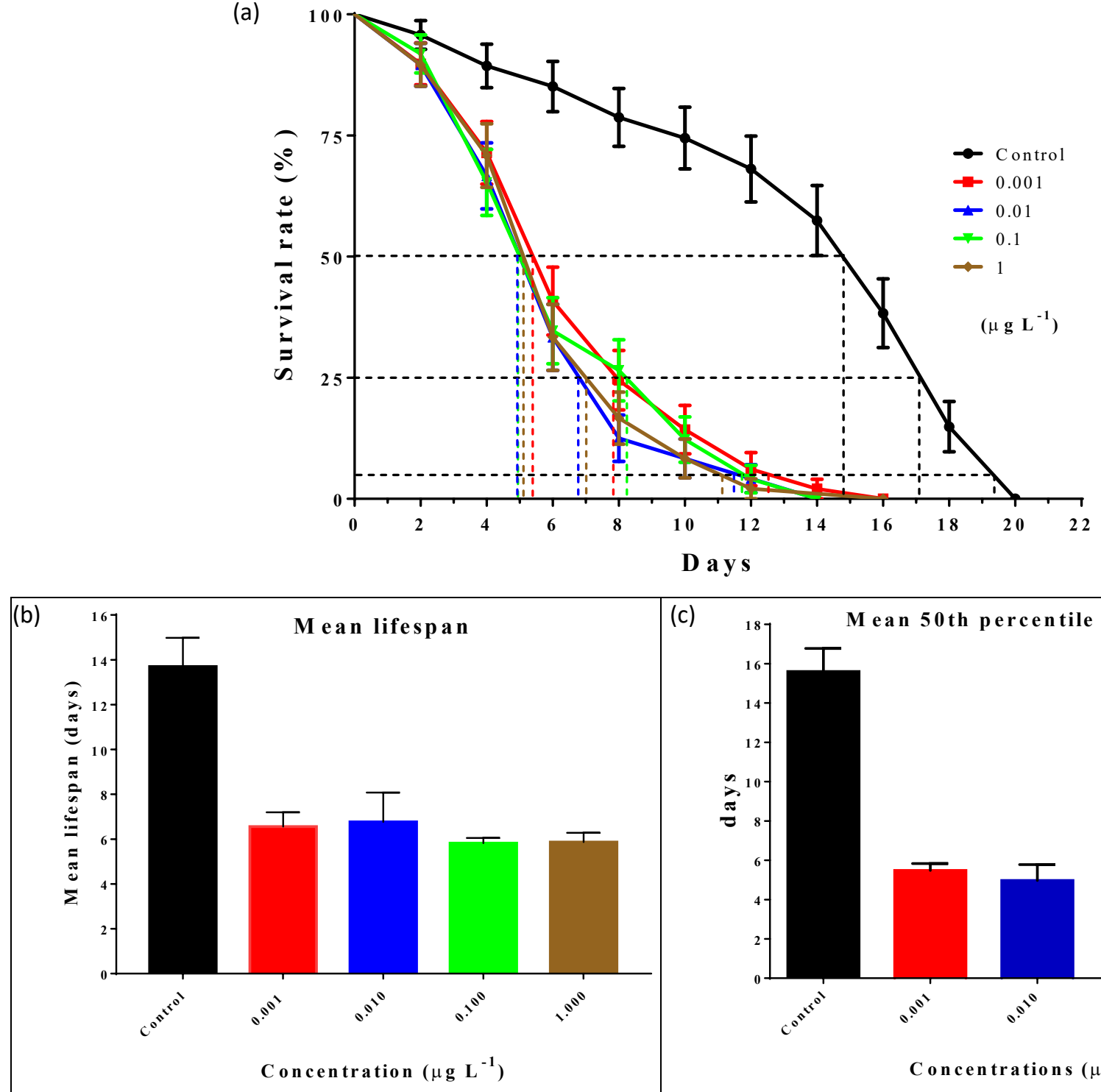

(c)

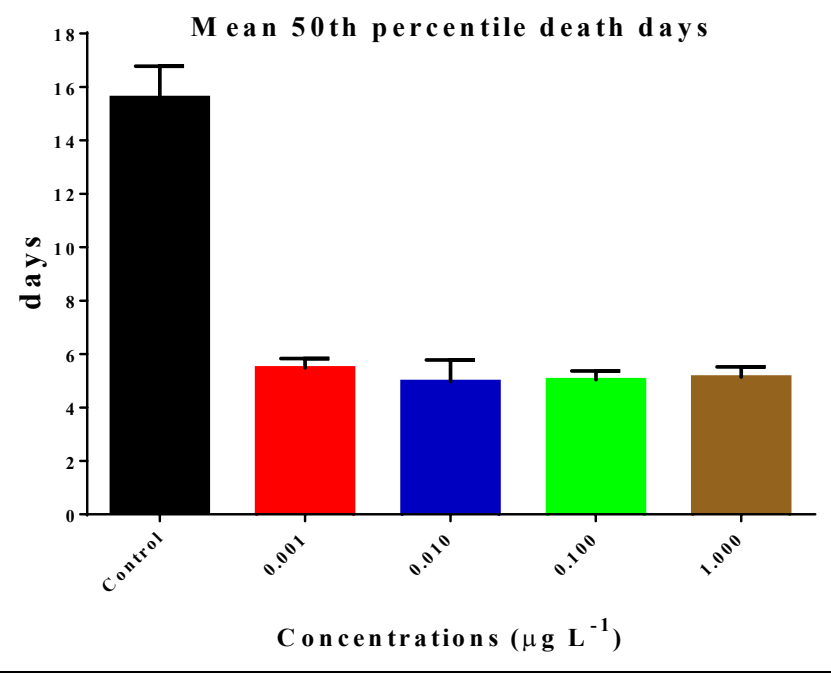

(d)
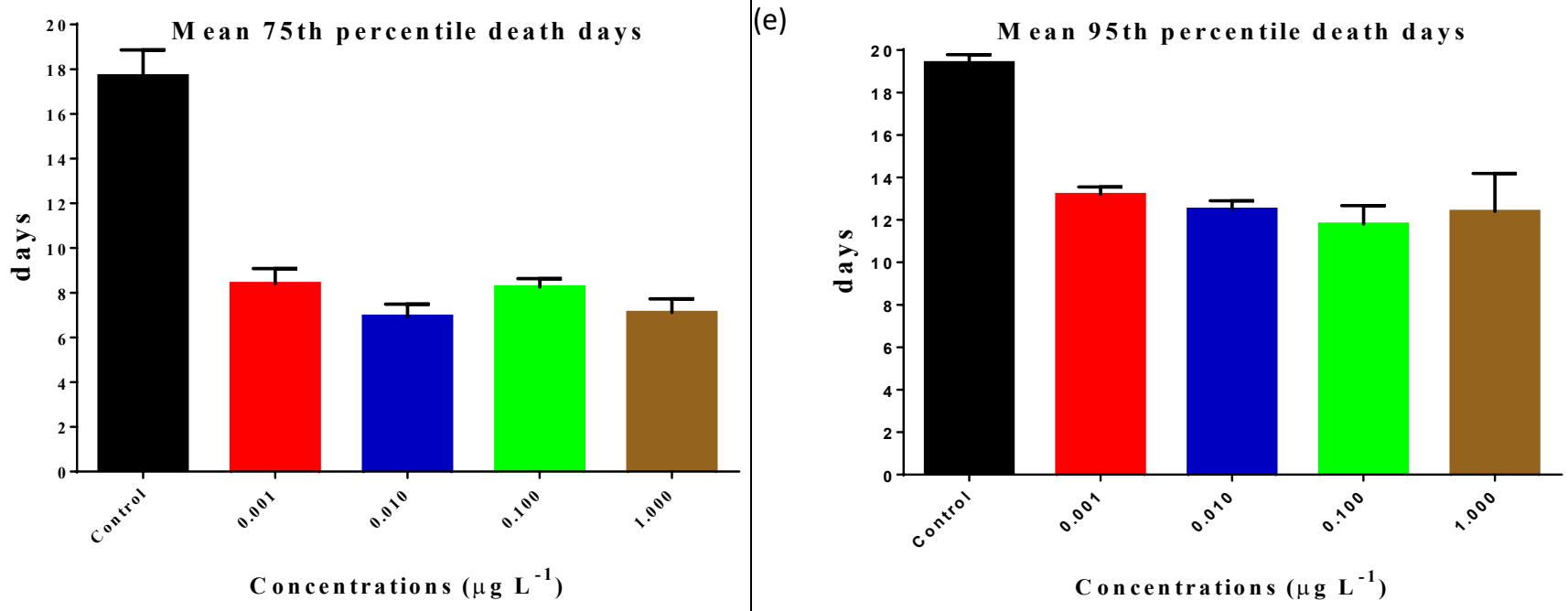

Fig. 4. Lifespan of $C$. elegans after prolonged exposure to GO NPs at levels of $0.00100,0.0100,0.100$, and $1.00 \mu \mathrm{L}^{-1}$ and the control (a) nanotoxic assessment of worms with prolonged GO exposure for lifespan, (b) mean lifespan, (c) mean day of $50^{\text {th }}$ percentile death, (d) mean day of $75^{\text {th }}$ percentile death, and (e) mean day of $95^{\text {th }}$ percentile death. Bars shown as mean \pm SD. Significant differences were expressed as $* p<0.05, * * p<0.01$, and $* * * p<0.001$. 
prolonged exposure to $0.00100,0.100$, and $1.00 \mu \mathrm{g} \mathrm{L}^{-1} \mathrm{GO} N \mathrm{NP}$ compared with the untreated control are shown in Fig. 5. The activated expressions of sod-1, sod-3, and ctl-2 at the concentrations of $0.00100,0.100$, and $1.00 \mu \mathrm{g} \mathrm{L}^{-1}$ in the GO NP-exposed C. elegans were significantly higher than those in the untreated control. SOD is a key enzyme in the detoxification function of free radicals. It removed free radicals generated from GO NPs in extracellular sources in nematodes. Results similar to those found in the present study were also found in previous studies (Wu et al., 2013; Zhao et al., 2016b), which indicates that GO NPs could induce sod-1 or sod-3 activation. The findings from Wu's study suggested that oxidative stress induced in the treated GO NP nematodes may be related to changes of SOD activities (Wu et al., 2013). Based on these findings, it can be inferred that oxidative stress is a possible mechanism causing adverse effects on neurodevelopment and neurobehavioral development after prolonged GO NP exposure, as suggested in previous reports (Wu et al., 2013; Zhao et al., 2016b), in combination with the results of induced SOD activation and neurotoxicity in the GO-exposed nematodes in the present study (Figs. 3 and 5). Furthermore, sod-1, sod-3, and ctl-2 activation may be associated with the shortened longevity in the GO-exposed worms, based on Figs. 4 and 5. In Zhou's study (Zhou et al., 2016), C. elegans ctl-2 gene encoded peroxisomal catalase was linked to environmental oxidative stress after worms were exposed to bisphenol A. Few studies have addressed to link between ctl-2 expression and $\mathrm{GO}$ exposure in $C$. elegans. Although a positive association between ctl-2 expression and GO NP exposure was shown in the present study, the mechanism is still unclear.

Finally, it was concluded in the present study that extremely low doses of GO NPs, compared with the dosages discussed in recent published articles, can cause reproductive and neurobehavioral

a.

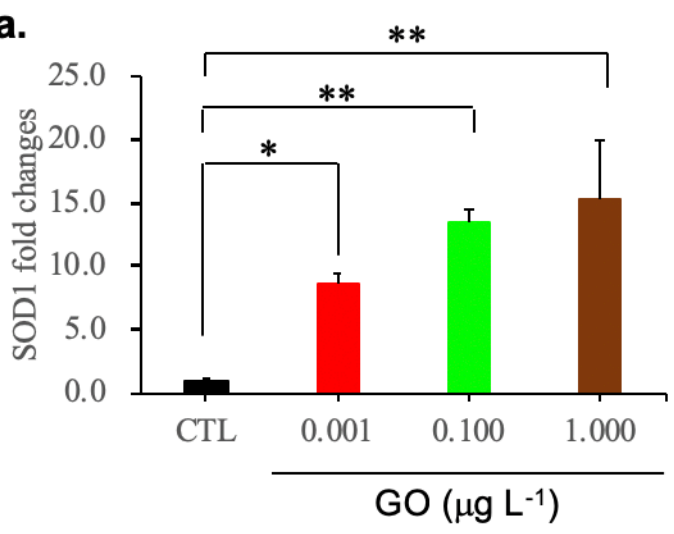

c.

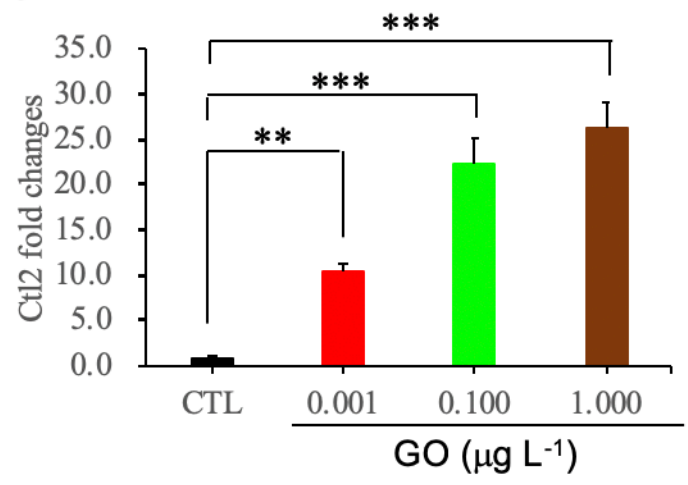

b.

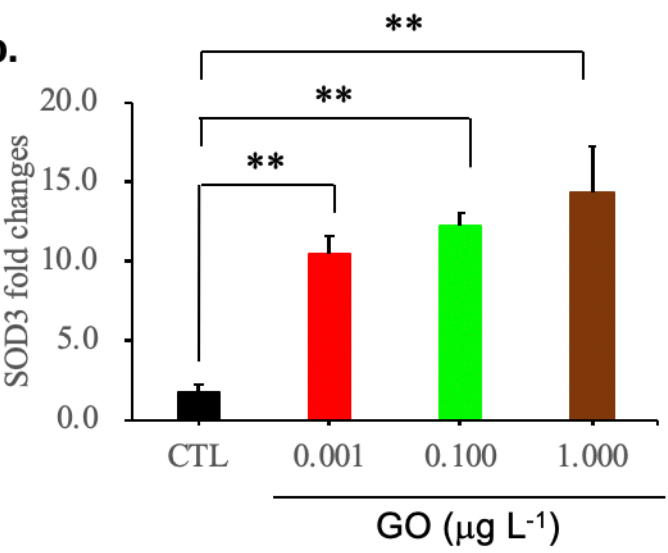

Fig. 5. Gene expression in C. elegans with prolonged exposure to GO NPs at the levels of the untreated control, $0.00100,0.100$, and $1.00 \mu \mathrm{g} \mathrm{L} \mathrm{L}^{-1}$ (a) SOD-1 (C15F1.7), (b) SOD-3 (C08A9.1), and (c) ctl-2 (Y54G11A.5); Actin-1 (T04C12.6) as the internal control. Bars shown as mean \pm SD. Significant differences were expressed as ${ }^{*} p<0.05,{ }^{* *} p<0.01$, and ${ }^{* * *} p<0.001$. 
toxicity and induce several-fold increases of sod-1, sod-3, and clt-2 gene expression. It is worth noting that in the present study, the potentially toxic effects of environmental levels of GO NPs in in-vivo $C$. elegans models were evaluated to show the negative impacts on reproduction, neurobehavioral development, and oxidative stress. It is thus reiterated that based on our findings, GO NPs at environmental levels may cause chronically toxic effects.

\section{CONCLUSIONS}

It is the first time to use the low dosage of GO NPs treating in the in-vivo model to find the adverse effects in nematodes. Based on our findings, prolonged exposure to GO NPs causes reproductive effects, generates neurotoxicity, shortens longevity, and induces oxidative stress in C. elegans. It is reiterated that low-dose GO NPs at environmental levels from 0.00100 to $1.00 \mu \mathrm{g} \mathrm{L}^{-1}$ caused significantly negative impacts on nematodes in contrast to the current published data. Thus, the adverse effects of low-level GO NPs on human health should be evaluated in the future.

\section{ACKNOWLEDGMENTS}

This study was supported by grants from the Ministry of Science and Technology (MOST 1062221-E-020-001-MY3) and Kaohsiung Veterans General Hospital, Pingtung Branch (VHCL108002). We acknowledge Ms. Danielle E. Que in the Department of Environmental engineering, National Cheng Kung University for assisting us to maintain, culture, and test $C$. elegans. We also want to thank Miss Yi-Jun Hsieh from Kaohsiung Medical University for assisting us in performing the C. elegans experiments. We would also like to thank Dr. Chang-Shi Chen at National Cheng Kung University for his advice and help in obtaining the $\mathrm{C}$. elegans culture.

\section{DISCLAIMER}

The authors of this paper declare no conflict of interest.

\section{REFERENCES}

Ahmed, F., Rodrigues, D.F. (2013). Investigation of acute effects of graphene oxide on wastewater microbial community: A case study. J. Hazard. Mater. 256-257, 33-39. https://doi.org/10.101 6/j.jhazmat.2013.03.064

Bengtson, S., Knudsen, K.B., Kyjovska, Z.O., Berthing, T., Skaug, V., Levin, M., Koponen, I.K., Shivayogimath, A., Booth, T.J., Alonso, B., Pesquera, A., Zurutuza, A., Thomsen, B.L., Troelsen, J.T., Jacobsen, N.R., Vogel, U. (2017). Differences in inflammation and acute phase response but similar genotoxicity in mice following pulmonary exposure to graphene oxide and reduced graphene oxide. PLoS One 12, e0178355. https://doi.org/10.1371/journal.pone.0178355

Bianco, A., Cheng, H.M., Enoki, T., Gogotsi, Y., Hurt, R.H., Koratkar, N., Kyotani, T., Monthioux, M., Park, C.R., Tascon, J.M.D., Zhang, J. (2013). All in the graphene family - A recommended nomenclature for two-dimensional carbon materials. Carbon 65, 1-6. https://doi.org/10.1016/ j.carbon.2013.08.038

Brenner, S. (1974). The genetics of Caenorhabditis elegans. Genetics 77, 71-94.

Chen, H., Li, H., Wang, D. (2017). Graphene oxide dysregulates Neuroligin/NLG-1-mediated molecular signaling in interneurons in Caenorhabditis elegans. Sci. Rep. 7, 41655. https://doi.org/10.1038/srep41655

Chen, Y., Hu, X., Sun, J., Zhou, Q. (2016a). Specific nanotoxicity of graphene oxide during zebrafish embryogenesis. 10, 42-52. https://doi.org/10.3109/17435390.2015.1005032

Chung, M.C., Tsai, M.H., Que, D.E., Bongo, S.J., Hsu, W.L., Tayo, L.L., Lin, Y.H., Lin, S.L., Gou, Y.Y., Hsu, Y.C., Hou, W.C., Huang, K.L., Chao, H.R. (2019). Fine particulate matter-induced toxic effects in an animal model of Caenorhabditis elegans. Aerosol Air Qual. Res. 19, 1068-1078. https://doi.org/10.4209/aaqr.2019.03.0127 
Chung, M.C., Huang, K.L., Avelino, J.L., Tayo, L.L., Lin, C.C., Tsai, M.H., Lin, S.L., Mansor, W.N.W., Su, C.K., Huang, S.T. (2020). Toxic assessment of heavily traffic-related fine particulate matter using an in-vivo wild-type Caenorhabditis elegans model. Aerosol Air Qual. Res. 20, 1974-1986. https://doi.org/10.4209/aaqr.2020.05.0192

Cole, R.D., Anderson, G.L., Williams, P.L. (2004). The nematode Caenorhabditis elegans as a model of organophosphate-induced mammalian neurotoxicity. Toxicol. Appl. Pharm. 194, 248-256. https://doi.org/10.1016/j.taap.2003.09.013

De Marchi, L., Pretti, C., Gabriel, B., Marques, P.A.A.P., Freitas, R., Neto, V. (2018). An overview of graphene materials: properties, applications and toxicity on aquatic environments. Sci. Total Environ. 631-632, 1440-1456. https://doi.org/10.1016/j.scitotenv.2018.03.132

Du, T., Adeleye, A.S., Keller, A.A., Wu, Z., Han, W., Wang, Y., Zhang, C., Li, Y. (2017). Photochlorination-induced transformation of graphene oxide: mechanism and environmental fate. Water Res. 124, 372-380. https://doi.org/10.1016/j.watres.2017.07.054

Fakhri, A. (2017). Adsorption characteristics of graphene oxide as a solid adsorbent for aniline removal from aqueous solutions: kinetics, thermodynamics and mechanism studies. J. Saudi Chem. Soc. 21, S52-S57. https://doi.org/10.1016/j.jscs.2013.10.002

Giust, D., Lucío, M.I., El-Sagheer, A.H., Brown, T., Williams, L.E., Muskens, O.L., Kanaras, A.G. (2018). Graphene oxide-upconversion nanoparticle based portable sensors for assessing nutritional deficiencies in crops. ACS Nano 12, 6273-6279. https://doi.org/10.1021/acsnano.8 b03261

Gonzalez-Moragas, L., Roig, A., Laromaine, A. (2015). C. elegans as a tool for in vivo nanoparticle assessment. Adv. Colloid Interface Sci. 219, 10-26. https://doi.org/10.1016/j.cis.2015.02.001

Goodwin, D.G., Adeleye, A.S., Sung, L., Ho, K.T., Burgess, R.M., Petersen, E.J. (2018). Detection and quantification of graphene-family nanomaterials in the environment. Environ. Sci. Technol. 52, 4491-4513. https://doi.org/10.1021/acs.est.7b04938

Guo, X., Mei, N. (2014). Assessment of the toxic potential of graphene family nanomaterials. J. Food Drug Anal. 22, 105-115. https://doi.org/10.1016/j.jfda.2014.01.009

He, K., Chen, G., Zeng, G., Peng, M., Huang, Z., Shi, J., Huang, T. (2017). Stability, transport and ecosystem effects of graphene in water and soil environments. Nanoscale 9, 5370-5388. https://doi.org/10.1039/C6NR09931A

Hunt, P.R. (2017). The C. elegans model in toxicity testing. J. Appl. Toxicol. 37, 50-59. https://doi.org/10.1002/jat.3357

Jamialahmadi, N., Safari, E., Baghdadi, M. (2018). Interaction of graphene oxide nano-sheets and landfill leachate bacterial culture. Environ. Technol. 39, 2457-2466. https://doi.org/10.1080/0 9593330.2017.1356875

Kang, S., Mauter, M.S., Elimelech, M. (2009). Microbial cytotoxicity of carbon-based nanomaterials: Implications for river water and wastewater effluent. Environ. Sci. Technol. 43, 2648-2653. https://doi.org/10.1021/es8031506

Kim, M., Eom, H.J., Choi, I., Hong, J., Choi, J. (2020). Graphene oxide-induced neurotoxicity on neurotransmitters, AFD neurons and locomotive behavior in Caenorhabditis elegans. Neurotoxicology 77, 30-39. https://doi.org/10.1016/j.neuro.2019.12.011

Kim, Y., Jeong, J., Yang, J., Joo, S.W., Hong, J., Choi, J. (2018). Graphene oxide nano-bio interaction induces inhibition of spermatogenesis and disturbance of fatty acid metabolism in the nematode Caenorhabditis elegans. Toxicology 410, 83-95. https://doi.org/10.1016/j.tox.2018. 09.006

Konios, D., Stylianakis, M.M., Stratakis, E., Kymakis, E. (2014). Dispersion behaviour of graphene oxide and reduced graphene oxide. J. Colloid Interface Sci. 430, 108-112. https://doi.org/10.10 16/j.jcis.2014.05.033

Li, F., Jiang, X., Zhao, J., Zhang, S. (2015). Graphene oxide: A promising nanomaterial for energy and environmental applications. Nano Energy 16, 488-515. https://doi.org/10.1016/j.nanoen. 2015.07.014

Li, M., Zhu, J., Wang, M., Fang, H., Zhu, G., Wang, Q. (2019). Exposure to graphene oxide at environmental concentrations induces thyroid endocrine disruption and lipid metabolic disturbance in Xenopus laevis. Chemosphere 236: 124834. https://doi.org/10.1016/j.chemosp here.2019.124834

Li, Y., Ma, R., Liu, X., Qi, Y., Abulikemu, A., Zhao, X., Duan, H., Zhou, X., Guo, C., Sun, Z. (2019). 
Endoplasmic reticulum stress-dependent oxidative stress mediated vascular injury induced by silica nanoparticles in vivo and in vitro. Nanolmpact 14, 100169. https://doi.org/10.1016/j.imp act.2019.100169

Liu, P., Shao, H., Kong, Y., Wang, D. (2020). Effect of graphene oxide exposure on intestinal Wnt signaling in nematode Caenorhabditis elegans. J. Environ. Sci. 88, 200-208. https://doi.org/10. 1016/j.jes.2019.09.002

Liu, X.T., Mu, X.Y., Wu, X.L., Meng, L.X., Guan, W.B., Ma, Y.Q., Sun, H., Wang, C.J., Li, X.F. (2014). Toxicity of multi-walled carbon nanotubes, graphene oxide, and reduced graphene oxide to zebrafish embryos. Biomed. Environ. Sci. 27, 676-683. https://doi.org/10.3967/bes2014.103

Lyon, D.Y., Alvarez, P.J.J. (2008). Fullerene water suspension ( $\left.\mathrm{Nc}_{60}\right)$ exerts antibacterial effects via ROS-independent protein oxidation. Environ. Sci. Technol. 42, 8127-8132. https://doi.org/10. 1021/es801869m

Maharubin, S., Zhang, X., Zhu, F., Zhang, H.C., Zhang, G., Zhang, Y. (2016). Synthesis and applications of semiconducting graphene. J. Nanomater. 2016, 6375962. https://doi.org/10.1 155/2016/6375962

Pan, Y., Sahoo, N.G., Li, L. (2012). The application of graphene oxide in drug delivery. Expert Opin. Drug Deliv. 9, 1365-1376. https://doi.org/10.1517/17425247.2012.729575

Patlolla, A.K., Rondalph, J., Tchounwou, P.B. (2017). Biochemical and histopathological evaluation of graphene oxide in sprague-dawley rats. Austin J. Environ. Toxicol. 3, 1021.

Pelin, M., Fusco, L., Martín, C., Sosa, S., Frontiñán-Rubio, J., González-Domínguez, J.M., DuránPrado, M., Vázquez, E., Prato, M., Tubaro, A. (2018). Graphene and graphene oxide induce ROS production in human HaCaT skin keratinocytes: the role of xanthine oxidase and NADH dehydrogenase. Nanoscale 10, 11820-11830. https://doi.org/10.1039/C8NR02933D

Piechulek, A., von Mikecz, A. (2018). Life span-resolved nanotoxicology enables identification of age-associated neuromuscular vulnerabilities in the nematode Caenorhabditis elegans. Environ. Pollut. 233: 1095-1103. https://doi.org/10.1016/j.envpol.2017.10.012

Prasad, C., Liu, Q., Tang, H., Yuvaraja, G., Long, J., Rammohan, A., Zyryanov, G.V. (2020). An overview of graphene oxide supported semiconductors based photocatalysts: Properties, synthesis and photocatalytic applications. J. Mol. Liq. 297, 111826. https://doi.org/10.1016/j. molliq.2019.111826

Qu, G., Liu, S., Zhang, S., Wang, L., Wang, X., Sun, B., Yin, N., Gao, X., Xia, T., Chen, J.J., Jiang, G.B. (2013a). Graphene oxide induces toll-like receptor 4 (TLR4)-dependent necrosis in macrophages. ACS Nano 7, 5732-5745. https://doi.org/10.1021/nn402330b

Qu, G., Wang, X., Liu, Q., Liu, R., Yin, N., Ma, J., Chen, L., He, J., Liu, S., Jiang, G. (2013b). The ex vivo and in vivo biological performances of graphene oxide and the impact of surfactant on graphene oxide's biocompatibility. J. Environ. Sci. 25, 873-881. https://doi.org/10.1016/S10010742(12)60252-6

Qu, M., Li, Y., Wu, Q., Xia, Y., Wang, D. (2017). Neuronal ERK signaling in response to graphene oxide in nematode Caenorhabditis elegans. Nanotoxicology 11, 520-533. https://doi.org/10.1 080/17435390.2017.1315190

Qu, M., Kong, Y., Yuan, Y., Wang, D. (2019). Neuronal damage induced by nanopolystyrene particles in nematode caenorhabditis elegans. Environ. Sci. Nano 6, 2591-2601. https://doi.org/10.1039/C9EN00473D

Ren, M., Zhao, L., Ding, X., Krasteva, N., Rui, Q., Wang, D. (2018). Developmental basis for intestinal barrier against the toxicity of graphene oxide. Part. Fibre Toxicol. 15, 26. https://doi.org/10.1186/s12989-018-0262-4

Rive, C., Reina, G., Wagle, P., Treossi, E., Palermo, V., Bianco, A., Delogu, L.G., Rieckher, M. Schumacher, B. (2019). Improved biocompatibility of amino-functionalized graphene oxide in Caenorhabditis elegans. Small 15, e1902699. https://doi.org/10.1002/smll.201902699

Rodrigues, D.F., Elimelech, M. (2010). Toxic effects of single-walled carbon nanotubes in the development of E. coli biofilm. Environ. Sci. Technol. 44, 4583-4589. https://doi.org/10.1021/ es1005785

Sanchez, V.C., Jachak, A., Hurt, R.H., Kane, A.B. (2012). Biological interactions of graphene-family nanomaterials: An interdisciplinary review. Chem. Res. Toxicol. 25, 15-34. https://doi.org/10.1 021/tx200339h

Souza, J.P., Baretta, J.F., Santos, F., Paino, I.M.M., Zucolotto, V. (2017). Toxicological effects of 
graphene oxide on adult zebrafish (Danio rerio). Aquat. Toxicol. 186, 11-18. https://doi.org/1 0.1016/j.aquatox.2017.02.017

Suárez-Iglesias, O., Collado, S., Oulego, P., Díaz, M. (2017). Graphene-family nanomaterials in wastewater treatment plants. Chem. Eng. J. 313, 121-135. https://doi.org/10.1016/j.cej.2016. 12.022

Tang, Z., Zhao, L., Yang, Z., Liu, Z., Gu, J., Bai, B., Liu, J., Xu, J., Yang, H. (2018). Mechanisms of oxidative stress, apoptosis, and autophagy involved in graphene oxide nanomaterial antiosteosarcoma effect. Int. J. Nanomed. 13, 2907-2919. https://doi.org/10.2147/IJN.S159388

Upadhyay, R.K., Soin, N., Roy, S.S. (2014). Role of graphene/metal oxide composites as photocatalysts, adsorbents and disinfectants in water treatment: A review. RSC Adv. 4, 38233851. https://doi.org/10.1039/C3RA45013A

Wu, Q., Wang, W., Li, Y., Li, Y., Ye, B., Tang, M., Wang, D. (2012). Small sizes of $\mathrm{TiO}_{2}-\mathrm{NPs}$ exhibit adverse effects at predicted environmental relevant concentrations on nematodes in a modified chronic toxicity assay system. J. Hazard. Mater. 243, 161-168. https://doi.org/10.10 16/j.jhazmat.2012.10.013

Wu, Q., Yin, L., Li, X., Tang, M., Zhang, T., Wang, D. (2013). Contributions of altered permeability of intestinal barrier and defecation behavior to toxicity formation from graphene oxide in nematode Caenorhabditis elegans. Nanoscale 5, 9934-9943. https://doi.org/10.1039/C3NR02 084C

Wu, Q., Zhao, Y., Li, Y., Wang, D., Wu, Q., Zhao, Y., Li, Y., Wang, D. (2014). Molecular signals regulating translocation and toxicity of graphene oxide in the nematode Caenorhabditis elegans. Nanoscale 6, 11204-11212. https://doi.org/10.1039/C4NR02688H

Wu, T., Xu, H., Liang, X., Tang, M. (2019). Caenorhabditis elegans as a complete model organism for biosafety assessments of nanoparticles. Chemosphere 221, 708-726. https://doi.org/10.10 16/j.chemosphere.2019.01.021

Yan, H., Tao, X., Yang, Z., Li, K., Yang, H., Li, A.M., Cheng, R. (2014). Effects of the oxidation degree of graphene oxide on the adsorption of methylene blue. J. Hazard. Mater. 268, 191-198. https://doi.org/10.1016/j.jhazmat.2014.01.015

Yang, K., Gong, H., Shi, X., Wan, J., Zhang, Y., Liu, Z. (2013). In vivo biodistribution and toxicology of functionalized nano-graphene oxide in mice after oral and intraperitoneal administration. Biomaterials 34, 2787-2795. https://doi.org/10.1016/j.biomaterials.2013.01.001

Yang, X., Qin, J., Jiang, Y., Chen, K., Yan, X., Zhang, D., Li, R., Tang, H. (2015). Fabrication of $\mathrm{P} 25 / \mathrm{Ag}_{3} \mathrm{PO}_{4} /$ graphene oxide heterostructures for enhanced solar photocatalytic degradation of organic pollutants and bacteria. Appl. Catal., B 166-167, 231-240. https://doi.org/10.1016/ j.apcatb.2014.11.028

Zhang, S., Yang, K., Feng, L., Liu, Z. (2011). In vitro and in vivo behaviors of dextran functionalized graphene. Carbon 49: 4040-4049. https://doi.org/10.1016/j.carbon.2011.05.056

Zhang, W., Wang, C., Li, Z., Lu, Z., Li, Y., Yin, J., Zhou, Y., Gao, X., Fang, Y., Nie, G., Zhao, Y. (2012). Unraveling stress-induced toxicity properties of graphene oxide and the underlying mechanism. Adv. Mater. 24, 5391-5397. https://doi.org/10.1002/adma.201202678

Zhang, X., Zhou, Q., Zou, W., Hu, X. (2017). Molecular mechanisms of developmental toxicity induced by graphene oxide at predicted environmental concentrations. Environ. Sci. Technol. 51, 7861-7871. https://doi.org/10.1021/acs.est.7b01922

Zhao, J., Wang, Z., White, J.C., Xing, B. (2014). Graphene in the aquatic environment: Adsorption, dispersion, toxicity and transformation. Environ. Sci. Technol. 48, 9995-10009. https://doi.org/ 10.1021/es5022679

Zhao, Y., Wu, Q., Wang, D. (2016a) An epigenetic signal encoded protection mechanism is activated by graphene oxide to inhibit its induced reproductive toxicity in Caenorhabditis elegans. Biomaterials 79, 15-24. https://doi.org/10.1016/j.biomaterials.2015.11.052

Zhao, Y., Yang, R., Rui, Q., Wang, D. (2016b). Intestinal insulin signaling encodes two different molecular mechanisms for the shortened longevity induced by graphene oxide in Caenorhabditis elegans. Sci. Rep. 6, 24024. https://doi.org/10.1038/srep24024

Zhao, Y., Zhi, L., Wu, Q., Yu, Y., Sun, Q., Wang, D. (2016c). p38 MAPK-SKN-1/Nrf signaling cascade is required for intestinal barrier against graphene oxide toxicity in Caenorhabditis elegans. Nanotoxicology 10, 1469-1479. https://doi.org/10.1080/17435390.2016.1235738

Zhao, Y., Chen, H., Yang, Y., Wu, Q., Wang, D. (2020). Graphene oxide disrupts the protein-protein 
interaction between Neuroligin/NLG-1 and DLG-1 or MAGI-1 in nematode Caenorhabditis elegans. Sci. Total Environ. 700, 134492. https://doi.org/10.1016/j.scitotenv.2019.134492

Zhou, D., Yang, J., Li, H., Lu, Q., Liu, Y.D., Lin, K.F. (2016). Ecotoxicity of bisphenol A to Caenorhabditis elegans by multigenerational exposure and variations of stress response in vivo across generations. Environ. Pollut. 208, 767-773. https://doi.org/10.1016/j.envpol.2015.10.057 